\title{
Macroscopic brain architecture changes and white matter pathology in acromegaly: a clinicoradiological study
}

\author{
C. Sievers · P. G. Sämann · T. Dose $\cdot$ C. Dimopoulou • \\ D. Spieler · J. Roemmler $\cdot$ J. Schopohl $\cdot$ M. Mueller . \\ H. J. Schneider · M. Czisch · H. Pfister · G. K. Stalla
}

Published online: 3 October 2008

(c) The Author(s) 2008. This article is published with open access at Springerlink.com

\begin{abstract}
Although long-term exposure of the brain to increased GH/IGF-1 likely influences cerebral functions, no in vivo studies have been directed towards changes of the brain structure in acromegaly. Here, we used high resolution magnetic resonance images to compare volumes of gray matter (GM), white matter (WM) and cerebrospinal fluid (CSF) of forty-four patients with acromegaly to an age and gender matched, healthy control group $(n=44)$. In addition, white matter lesions (WMLs) were quantified and graded. Patients exhibited larger GM $(+3.7 \%$ compared with controls, $P=0.018$ ) and WM volumes $(+5.1 \%, P=0.035)$ at the expense of CSF. Differences of WML counts between patients and controls were subtle, however, showing more patients in the 21-40 lesions
\end{abstract}

C. Sievers and P. G. Sämann have contributed equally to this work.

C. Sievers $(\bowtie) \cdot$ C. Dimopoulou · H. J. Schneider · G. K. Stalla

Department of Endocrinology, Max Planck Institute of

Psychiatry, Kraepelinstr. 2-10, 80804 Munich, Germany

e-mail: csievers@mpipsykl.mpg.de

P. G. Sämann · M. Czisch

Department of Magnetic Resonance Imaging, Max Planck Institute of Psychiatry, Kraepelinstr. 2-10, 80804 Munich, Germany

T. Dose $\cdot$ D. Spieler $\cdot$ M. Mueller

Department of Endocrinology and Department of Magnetic

Resonance Imaging, Max Planck Institute of Psychiatry,

Kraepelinstr. 2-10, 80804 Munich, Germany

J. Roemmler · J. Schopohl · H. J. Schneider

Department of Internal Medicine, Department of Endocrinology,

Ludwig-Maximilians-Universität, Munich, Germany

H. Pfister

Department of Molecular Psychology, Max Planck Institute of Psychiatry, Kraepelinstr. 2-10, 80804 Munich, Germany category $(P=0.044)$. In conclusion, this MRI study provides first evidence that acromegalic patients exhibit disturbances of the macroscopic brain tissue architecture. Furthermore, acromegalic patients may have an increased risk of neurovascular pathology, likely due to secondary metabolic and vascular comorbidities.

Keywords Acromegaly - Insulin-like-growth factor 1 . Growth hormone - White matter lesions · Brain volume . MRI

\section{Introduction}

Acromegaly is characterized by excessive levels of growth hormone (GH) and insulin-like-growth factor 1 (IGF-1) that are caused by pituitary adenomas in most cases. The clinical condition is associated with a vast range of complications and comorbidities such as organomegaly, skeletal deformations with secondary arthropathy, diabetes mellitus, arterial hypertension, secondary pituitary deficiency, and neurological complications such as nerve compression syndromes [1]. With regard to psychiatric syndromes, both personality changes and a psychopathological risk profile with an increased prevalence of affective disorders have been suspected [2-7].

The increasing attention for neuropsychiatric aspects of acromegaly is concurring with preclinical research that has gained insight into the role of GH and IGF-1 in brain function. Both factors are involved in the development of the brain [8-10]. More specifically, GH and IGF-1 play a part in the regulation of brain growth including neuronal cell proliferation, differentiation and myelination during development [11-13]. Further, both postnatally and in the adult rodent, IGF-1 promotes neurogenesis and synaptogenesis in 
the hippocampal dentate $[13,14]$. In turn, the experimental disruption of IGF-1 gene function results in reduced brain size, CNS hypomyelination and loss of hippocampal granule and striatal parvalbumin-containing neurons [15].

While many of the effects of IGF-1 and GH in the brain are mediated by locally produced hormones, also circulating hormones can reach the brain after crossing or bypassing the blood brain barrier [16]. In acromegaly, from 6 to 10 years of hormone excess passes until clinical signs or symptoms of acromegaly lead to the diagnostic work-up and treatment [17]. Such long-lasting exposure to IGF-1 and GH interferes with regulation of trophic processes in many organ systems [1], and may be supposed to affect brain function and structure. Here, magnetic resonance imaging (MRI) provides a number of techniques that allow a non-invasive characterization of neuropathological changes, yet no quantitative analyses of brain changes in acromegaly have been presented so far.

We investigated if the GH and IGF-1 excess in acromegalic patients alters the macroscopic structure of the brain as determined by MRI. More specifically, we gained volumes of the main compartments of the brain-gray matter (GM), white matter (WM), and cerebrospinal fluid (CSF)—from high resolution MRI. The GM compartment includes the whole cortex and the deep nuclei, whereas the WM compartment represents the entirety of the fiber tracts. In addition, we explored if acromegalic patients show MRI signs of neurovascular pathology that may add to their neuropsychiatric comorbidity.

\section{Methods}

Subject recruitment and clinical characterization

Between 04/2006 and 07/2007, 44 patients with previously diagnosed acromegaly were recruited from the endocrine outpatient unit of the Max Planck Institute of Psychiatry (MPIP), Munich, and the Department of Internal Medicine, Ludwig-Maximilians-Universität, Munich. All patients gave their written informed consent to participate in the study. Patients received a comprehensive medical interview with regard to initial symptomatology, disease development, tumor characteristics, previous and current disease specific therapy, and current complaints. After exploration of their past medical history and systematic inquiry on comorbidities such as cardiovascular diseases, metabolic features, respiratory features, bone and joint features, malignancies, and secondary endocrine complications, patients were examined.

Additionally, basal hormonal levels and metabolic parameters were obtained in a fasting state at the day of study participation (between 8.00 and $10.00 \mathrm{am}$ ). Coronary artery disease, type 2 diabetes and dyslipidemia were recorded from physician's diagnoses, intake of respective medications, or abnormal laboratory values from patient's records. In the case of missing data or uncertainty, additional information was obtained by file review. Diagnoses of somatic comorbidities were made according to the respective guidelines. Serum concentrations of GH were measured using the automated Advantage chemiluminescent assay system (Nichols Diagnostics Institute, Bad Vilbel, Germany). IGF-1 was measured by automated chemiluminescent assays (IMMULITE 2000). Biochemical control was defined by (a) GH below $1 \mu \mathrm{g} / \mathrm{l}$ during a glucose tolerance test over $2 \mathrm{~h}$, and (b) IGF-1 level within two standard deviations of an age- and gender-adjusted normative sample [18]. IGF-1 values are indicated as a multiple of the upper limit of normal (xULN), calculated as follows: $x \mathrm{ULN}_{\mathrm{IGF}-1}=$ IGF-1 value/95th percentile of the age and gender adjusted normal range.

We further introduced a parameter "time of hormonal excess" aimed to inform on the time the patient has been exposed to increased IGF-1 or GH. It was calculated from the time of diagnosis until the date of MRI, augmented by an estimated 8 years of subclinically increased hormone levels as reported [17], and corrected for the time since documented biochemical control.

Forty-four age and gender matched controls were selected case-by-case from a database of 200 neuropsychiatrically healthy control subjects that were recruited and characterized at the MPIP. Medical comorbidities such as arterial hypertension, dyslipidemia and diabetes, as well as alcohol abuse and nicotine abuse were explored by questionnaires and standard laboratory tests.

Magnetic resonance image acquisition

The MRI protocol comprised axial fluid attenuated inversion recovery (FLAIR) images (TR 10,002 ms, TE $122.8 \mathrm{~ms}$, FOV $24 \times 24 \mathrm{~cm}^{2}$, in-plane resolution $0.9375 \times$ $1.071 \mathrm{~mm}^{2}, 20$ slices, slice thickness $5 \mathrm{~mm}$ ), axial proton density and T2-weighted double echo sequence (spin echo, TR 6,540 ms, TE 20.7/113.8 ms, FOV $24 \times 24 \mathrm{~cm}^{2}$, inplane resolution $0.5357 \times 0.5357 \mathrm{~mm}^{2}, 20$ slices, slice thickness $3 \mathrm{~mm}$ ), and sagittal T1 weighted high resolution image (spin echo, TR $9.7 \mathrm{~ms}$, TE $2.1 \mathrm{ms,} \mathrm{FOV}$ $25 \times 25 \mathrm{~cm}^{2}$, in-plane resolution $0.875 \times 0.875 \mathrm{~mm}^{2}$, 124-132 slices, slice thickness 1.2 mm; Fig. 1a).

Radiological assessment of white matter integrity

Rating of white matter lesions (WML) was performed on axial FLAIR images by two raters experienced in neuroradiological film reading (Fig. 1b). Both readers were blind for the main diagnosis, age and comorbidities of the subject 
Fig. 1 Examples of raw images and segmentation into GM, WM and CSF. a Example of high resolution T1-weighted image in sagittal view. b Typical multifocal white matter lesions, apparent as hyperintense areas on a FLAIR image of a study patient. c-e Exemplary axial slice of GM, WM and CSF of a patient after segmentation and spatial normalization. All maps were corrected for erronous inclusion of non-brain and non-CSF areas by applying a mask as overlaid in (e)
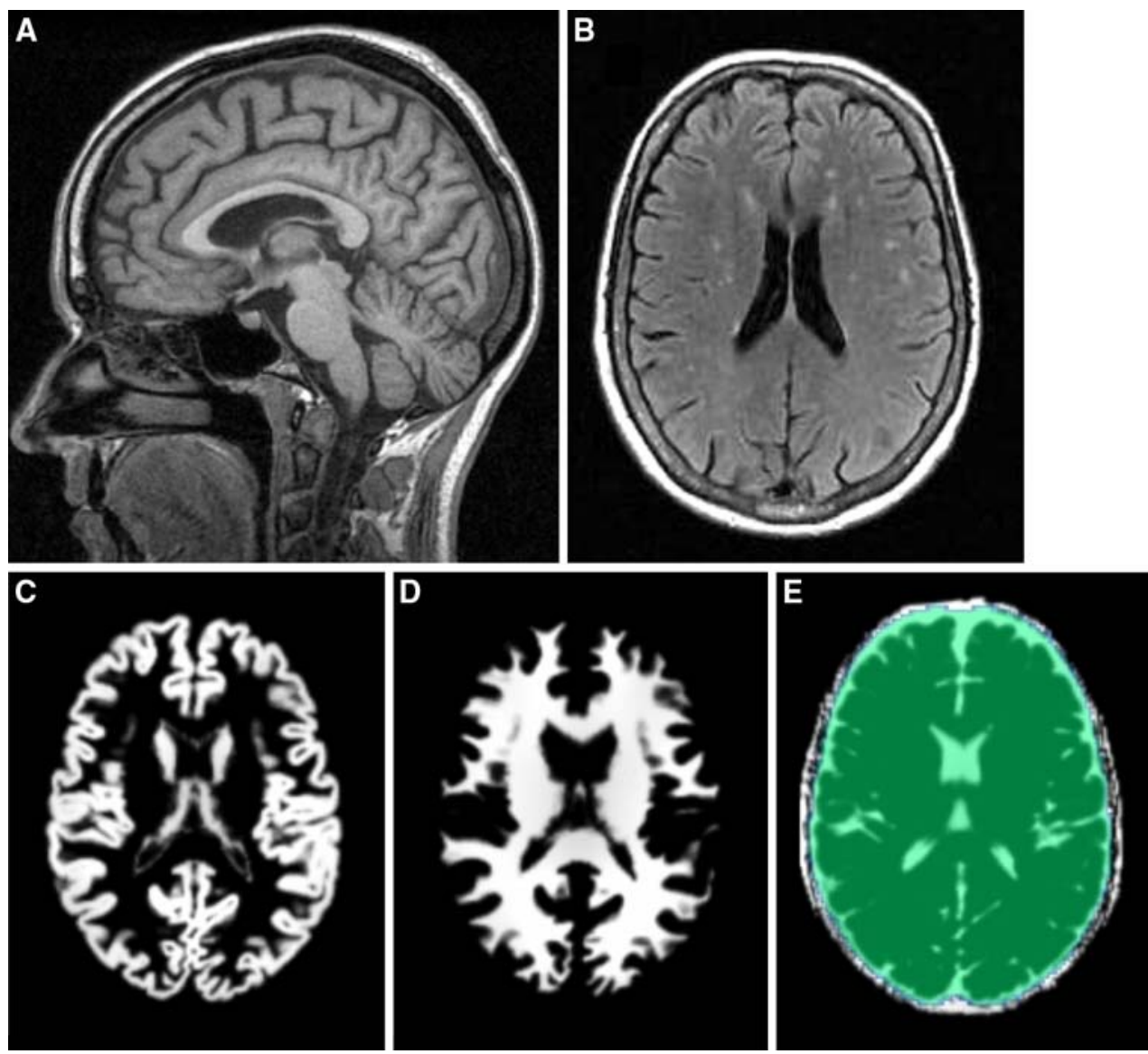

and worked independently from each other after a common training session. All axial FLAIR slices of a subject were screened simultaneously on a digital $5 \times 5$ panel, and focal WM hyperintensities larger than $2 \mathrm{~mm}$ were counted. Hyperintense bands adjacent to the anterior tip and posterior parts of the lateral ventricles as often present as normal variant, were disregarded. Lesions with continuous extension over two or more slices were counted as one lesion. After independent runs of each raters, all cases with discrepant lesion counts were re-assessed by both raters until consensus was achieved. In ambiguous cases of focal hyperintensities, proton density and axial T2 weighted images were consulted in addition. Due to the skewed distribution of WML counts and the increasing degree of uncertainty with higher counts, subjects were classified into WML categories as follows: class 0 : no WML; class I: 1-5 lesions; class II: 6-10 lesions; class III: 11-20 lesions; class IV: 21-40 lesions; class V: more than 40 lesions.

\section{Brain tissue volumetry}

High resolution T1-weighted images were segmentated into GM, WM, and CSF using the Matlab based (Matlab
7.0.4, MathWorks, Natick, USA) statistical parametric mapping (http://www.fil.ion.ucl.ac. uk/spm, version SPM5) software package. The implemented algorithm uses the unified segmentation approach [19] to provide voxelwise maps of GM, WM, and CSF in normalized space. For segmentation, prior probability maps (resolution $2 \times 2 \times 2 \mathrm{~mm}^{3}$, standard SPM5 distribution) as based on the International Consortium for Brain Mapping maps ([ICBM], http://www.loni.ucla.edu/ ICBM), along with default processing parameters were employed. The volume for each tissue class $\left(\mathrm{GM}_{\text {total }}, \mathrm{WM}_{\text {total }}, \mathrm{CSF}_{\text {total }}\right)$ was calculated as total of voxel intensities of normalized and modulated volume maps after masking out non-brain and non-CSF voxels. This approach has recently been validated and proven to be robust towards influences from pathological WM changes [20]. For this, a mask from a large sample $(N>500)$ of equally processed images that matched the intracranial vault was used (Fig. 1c-e). The masking procedure particularly improves measurement accuracy of the CSF volume and consequently of the total intracranial volume (TIV, calculated as $\mathrm{GM}_{\text {total }}+\mathrm{WM}_{\text {to- }}$ tal $\left.+\mathrm{CSF}_{\text {total }}\right)$. In addition, volumes normalized to TIV were calculated $\left(\mathrm{GM}_{\text {norm }}, \mathrm{WM}_{\text {norm }}, \mathrm{CSF}_{\text {norm }}\right)$. 


\section{Statistical analysis}

Comparison of comorbidities and WML

The frequency of comorbid diagnoses and anthropometric measures were compared using Fisher's exact tests or $t$ tests, respectively. The percentage of subjects in each WML class was compared between the patient and control group using two-sided Pearson chi square tests. The analysis was repeated separately for patients with shorter and longer exposure to hormone excess.

Analysis of volumes of gray matter, white matter, cerebrospinal fluid, and total intracranial volume

$\mathrm{GM}_{\text {total }}, \mathrm{WM}_{\text {total }}, \mathrm{CSF}_{\text {total }}$, and TIV were compared between patients and controls using analysis of covariance (ANCOVA) with age and gender as covariates. As a large proportion of the variance of the brain's volume is determined the TIV, the analyses were repeated with $\mathrm{GM}_{\text {norm }}$, $\mathrm{WM}_{\text {norm }}$, and $\mathrm{CSF}_{\text {norm}}$.

Relation of volumetric measures to estimated disease duration and current hormonal status

To detect a relationship between the time of hormonal excess and volumetric changes, patients were stratified into subgroups according to a median (10.9 years) split. ANCOVA was performed using a three-level group factor (controls, patients with below median [subgroup I] and above median [subgroup II] time of hormonal excess) and age and gender as covariates. Dependent variables were absolute brain volume $\left(\mathrm{GM}_{\text {total }}+\mathrm{WM}_{\text {total }}\right), \mathrm{GM}_{\text {total }}, \mathrm{WM}_{\text {total }}, \mathrm{CSF}_{\text {total }}$, the respective normalized volumes, and TIV. Subgroups did not differ with regard to gender distribution (women/men 13/9 and $10 / 12, P=0.547$, Fisher's exact test) and mean age $(56.5 \pm 14.0$ years and $52.2 \pm 13.8$ years, $P=0.305$, $t$-test). Expectedly, the mean age at diagnosis was lower in subgroup II $(51.4 \pm 14.4$ and $42.5 \pm 13.1$ years, $P=0.037, t$-test). Covariation of absolute and normalized volumes of the whole brain, GM, WM and CSF, and TIV with the current hormonal status was investigated by (a) multiple regression of the volumetric parameters on age, gender and $x \mathrm{ULN}_{\mathrm{IGF}-1}$, and (b) by comparing patients with $(N=27)$ and without $(N=17)$ biochemical control at the time of MRI, covarying for age and gender. The statistical significance threshold was set to 0.05 .

Relation of brain measures with vascular comorbidity status

After obtaining the main finding of an association between acromegaly and brain volume, cardiovascular comorbidities found to be different in frequency between patients and controls (arterial hypertension and diabetes, see result section) were summarized to one factor, and two-factoral ANCOVA was performed to investigate if this comorbidity status or its interaction with acromegaly underlies these effects. Age and gender were used as covariates.

\section{Results}

Demographic and clinical characteristics

In brief, the average time between the diagnosis of acromegaly and MRI was $7.8 \pm 6.8$ years. The estimated time of hormone excess was $12.5 \pm 5.2$ years. Patients showed a higher frequency of arterial hypertension $(P=0.001)$ and diabetes $(P=0.002$, Fisher's exact tests) and larger waist circumference $(P<0.001)$ and BMI values $(P<0.001$, two sample $t$-tests) compared with controls (Table 1).

Patient/control comparison of volumetric measures

Patients exhibited about 3.7\% larger absolute GM volumes than controls $\left(627 \pm 78 \mathrm{ml}\right.$ vs. $605 \pm 84 \mathrm{ml}, F_{1,40}=$ $5.854, P=0.018$ ) and about $5.1 \%$ larger absolute WM volumes than controls $(460 \pm 63 \mathrm{ml}$ vs. $438 \pm 63 \mathrm{ml}$, $F_{1,40}=4.607, P=0.035$ ). Absolute CSF volume was decreased in patients by about $3.7 \%(407 \pm 76 \mathrm{ml}$ vs. $423 \pm 77 \mathrm{ml}$ ), and TIV was increased in patients by about $2 \%(1,494 \pm 156 \mathrm{ml}$ vs. $1,465 \pm 153 \mathrm{ml})$, yet, these differences were not significant $(P=0.185$ and $P=0.174$, respectively) (Fig. 2). A similar pattern were revealed for normalized volumes with the $\mathrm{CSF}_{\text {norm }}$ difference reaching significance $\left(\mathrm{GM}_{\text {norm }}: F_{1,40}=3.619, P=0.061, \mathrm{WM}_{\text {norm: }}\right.$ : $F_{1,40}=4.066, \quad P=0.046, \quad \mathrm{CSF}_{\text {norm }}: \quad F_{1,40}=6.365$, $P=0.014)$. In summary, this pattern forwarded increases of the GM and WM compartment with a volume gain of each compartment of about $22 \mathrm{ml}$ at the expense of the CSF spaces and accompanied by (non-significant) TIV increases.

Influence of the estimated time of hormonal excess

Analysis of absolute brain volumes between controls and patients with shorter (subgroup I) and longer (subgroup II) exposure to increased hormone levels revealed a significant main effect $\left(F_{2,39}=3.450, P=0.036\right)$. Post-hoc tests pointed to a difference between controls and subgroup I $(P=0.014$, Fig. 3). Subgroup II was interpositioned between the control group and subgroup I with non-significant differences to either group. A similar pattern emerged for $\mathrm{GM}\left(F_{2,39}=3.117, P=0.050\right.$; post-hoc comparison of control group vs. subgroup I: $P=0.022$ ) and $\mathrm{WM}\left(F_{2,39}=2.751, P=0.070\right.$; post-hoc comparison 
Table 1 Demographic and clinical characteristics of patients and controls a Student's $t$-test, two-sided

b Fisher's exact test, two-sided

\begin{tabular}{|c|c|c|c|}
\hline & Patients $(N=44)$ & Controls $(N=44)$ & $P$-value \\
\hline Age, years & $54.1(13.7)$ & $53.8(13.4)$ & $0.828^{\mathrm{a}}$ \\
\hline Gender distribution & $52 \%$ females & $52 \%$ females & $1.000^{\mathrm{b}}$ \\
\hline Age at diagnosis, years & $46.9(14.4)$ & N/A & N/A \\
\hline Years since biochemical control & $3.2(5.5)$ & N/A & N/A \\
\hline Estimated time of hormonal excess, years & $12.5(5.2)$ & N/A & N/A \\
\hline Biochemically controlled at study entry & $27(61 \%)$ & N/A & N/A \\
\hline \multicolumn{4}{|l|}{ Type of adenoma } \\
\hline Macroadenoma & $36(82 \%)$ & N/A & N/A \\
\hline Microademona & $4(9 \%)$ & N/A & N/A \\
\hline Not known & $4(9 \%)$ & N/A & N/A \\
\hline \multicolumn{4}{|l|}{ Therapy } \\
\hline Neurosurgery & $41(93 \%)$ & N/A & N/A \\
\hline Radiation & $6(14 \%)$ & N/A & N/A \\
\hline Medical therapy & $27(61 \%)$ & N/A & N/A \\
\hline Arrhythmia & $10(23 \%)$ & N/A & N/A \\
\hline Cardiomyopathy & $5(11 \%)$ & N/A & N/A \\
\hline Cerebrovascular diseases & $4(9 \%)$ & N/A & N/A \\
\hline Hypertension & $27(61 \%)$ & $11(25 \%)$ & $0.001^{\mathrm{b}}$ \\
\hline Coronary artery disease & $5(11 \%)$ & N/A & N/A \\
\hline Myocardial infarction & $1(2 \%)$ & N/A & N/A \\
\hline Arthralgia & $28(64 \%)$ & N/A & N/A \\
\hline Diabetes mellitus & $14(32 \%)$ & $2(5 \%)$ & $0.002^{\mathrm{b}}$ \\
\hline Pituitary deficiency & $23(52 \%)$ & N/A & N/A \\
\hline Sleep apnea & $16(36 \%)$ & N/A & N/A \\
\hline Disturbances of lipid metabolism & $19(43 \%)$ & $13(30 \%)$ & $0.186^{\mathrm{b}}$ \\
\hline Alcohol abuse & $10(23 \%)$ & $5(11 \%)$ & $0.166^{\mathrm{b}}$ \\
\hline Nicotine abuse & $24(55 \%)$ & $20(45 \%)$ & $0.394^{\mathrm{b}}$ \\
\hline \multicolumn{4}{|l|}{ Anthropometric measures } \\
\hline Height, $\mathrm{cm}$ & $173.1(11.7)$ & $170.3(9.2)$ & $0.199^{\mathrm{a}}$ \\
\hline Waist circumference, $\mathrm{cm}$ & $98.1(12.7)$ & $87.4(11.1)$ & $<0.001^{\mathrm{a}}$ \\
\hline Body Mass Index (BMI), kg/m² & $29.0(5.2)$ & $24.8(2.7)$ & $<0.001^{\mathrm{a}}$ \\
\hline
\end{tabular}

of control group vs. subgroup I: $P=0.022)$. For CSF and TIV the main effect remained non-significant $(P=0.111$ and $P=0.412$, respectively). Analysis of the normalized volumes revealed similar patterns for $\mathrm{GM}_{\text {norm }}$ and $\mathrm{WM}_{\text {norm, }}$ while the main effect for the comparison of $\mathrm{CSF}_{\text {norm }}$ was significant $\left(F_{2,39}=4.864, P=0.010\right)$, with post-hoc comparisons showing decreased $\mathrm{CSF}_{\text {norm }}$ comparing subgroup I with controls $(P=0.002)$, and a trend difference between subgroup I and II $(P=0.077)$.

Correlation with current hormonal status

No significant differences of $\mathrm{GM}_{\text {total }}, \mathrm{WM}_{\text {total }}$, $\mathrm{BV}_{\text {total }}$, $\mathrm{CSF}_{\text {total }}$ or TIV were found between biochemically controlled and non-controlled patients. In addition, multiple regression analysis of $\mathrm{GM}, \mathrm{WM}$ and $\mathrm{BV}$ showed no significant contribution of $\mathrm{xULN} \mathrm{NGF-1}_{\mathrm{IGF}}$.

White matter lesion analysis

An equal proportion of patients and controls (34.1\%) was classified into the lowest WML class (no lesions). A higher proportion of patients was found in WML class IV (21-40 lesions, $18.2 \%$ vs. $4.6 \%, P=0.044$, Pearson chi square test) (Fig. 4). This difference was also seen in a comparison of the controls with the subgroup of patients with longer exposure to hormone excess $(P=0.024)$, however not in the subgroup with shorter exposure $(P=0.188)$. No differences were seen with regard to the other WML categories. 


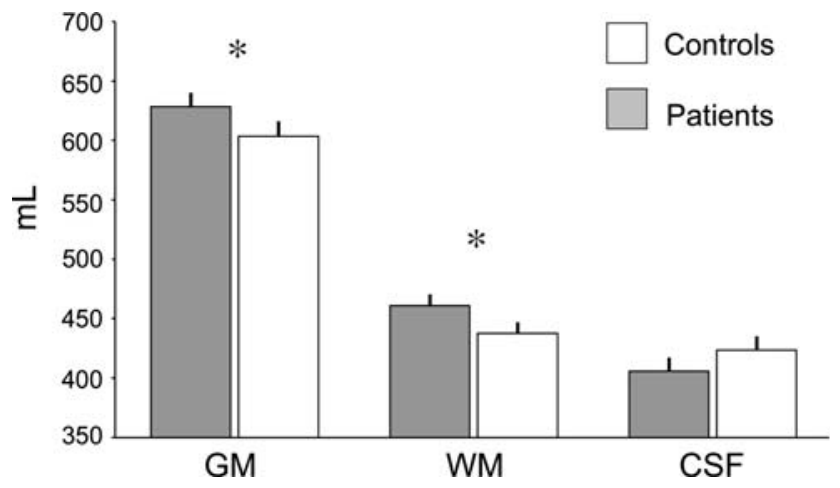

Fig. 2 Absolute volumes of gray matter, white matter and cerebrospinal fluid in acromegalic patients $(N=44)$ and control subjects $(N=44)$. Bars show estimated marginal means $(+\mathrm{SEM})$ corrected for age and gender, of the three compartments for patients and controls. GM: gray matter, WM: white matter, CSF: cerebrospinal fluid. *Significant differences for the group factor emerged for comparison of GM $(P=0.018)$ and WM $(P=0.035)$. CSF differences were significant for normalized, but not for absolute volumes (see result section)

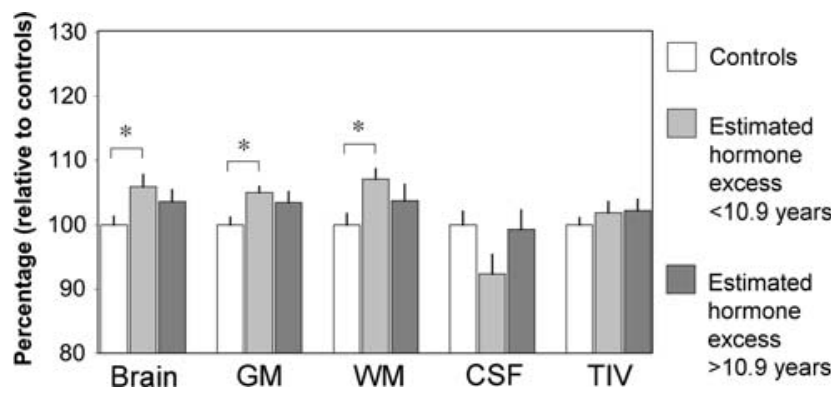

Fig. 3 Influence of the estimated time of hormone excess. Depiction of estimated marginal means (+SEM) after correction for age and gender effects, relative to mean values of the control group. Post-hoc comparisons revealed significant increases $(* P<0.05)$ in the patient subgroup I (below median 10.9 years of estimated hormone exposure) with regard to $\mathrm{GM}_{\text {total }}, \mathrm{WM}_{\text {total }}$ and absolute total brain volume. See result section for the analysis of normalized volumes

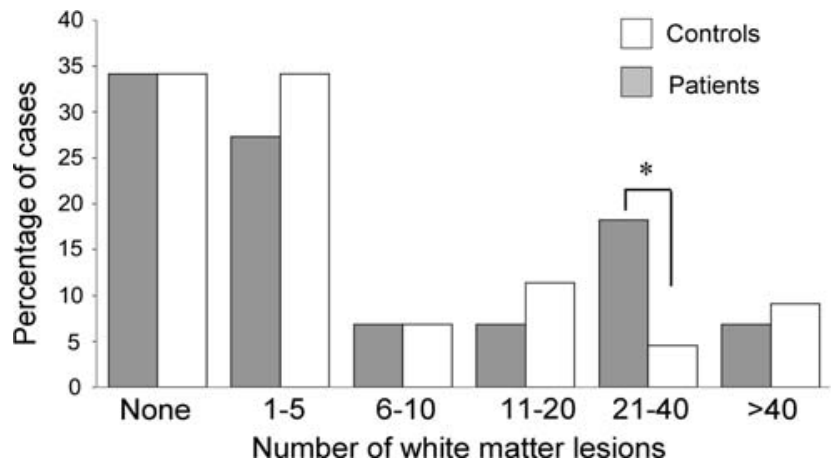

Fig. 4 White matter lesion counts. Relative frequencies of patients and controls in the six WML categories are given. Patients were significantly overrepresented in the $21-40$ WML class $(P=0.044$, Pearson chi square test)
Relation of brain measures to cardiovascular comorbidity status

The combined arterial hypertension/diabetes status variable was found to be associated with lower GM volume in patients and controls at trend level $(P=0.092)$, not interacting with the main diagnosis $(P=0.480)$. For WM and brain, only the main diagnosis (WM: $P=0.031$; brain: $P=0.007)$ but not the comorbidity status contributed significantly (WM: $P=0.533$; brain: $P=0.217$ ).

\section{Discussion}

Due to the widespread functions of IGF-1 and GH in many organ systems, acromegaly is a complex systemic disease. Similarly to other organ systems, the central nervous system may be affected by long-term exposure to increased levels of GH and IGF-1, however, no in vivo studies on brain structure (or metabolism) in acromegaly have been reported to our knowledge. In brief, the data from this study demonstrates that: (1) the excess of GH and IGF-1 is associated with a volumetric expansion of the GM $(+3.7 \%)$ and WM compartment $(+5.1 \%)$ of the brain, accompanied by compensatory CSF volume decreases and total intracranial volume increases, (2) these changes seem to occur during the first 10 years of hormone excess, and (3) patients exhibit more focal hyperintense WM lesions that served as marker of neurovascular pathology.

The volumetric expansion of GM and WM may be the result of the long-lasting exposure of neuronal or glial cells towards systemically elevated GH and IGF-1. Both factors are known to mediate the maturation of these cell types during brain development and continue to have pleiotropic effects in adulthood [10, 21]. IGF-1 overexpressionthough not directly comparable to systemically elevated IGF-1/GH levels - has been reported to lead to markedly increased brain size in mice [22], whereas IGF-1 knockout mice showed smaller brains [15]. These reports suggest that IGF-1 mediated trophic effects on the brain may indeed reach a macroscopic scale. It is not known, however, to what degree the altered brain phenotypes translated into behavioral changes or more generally, functional deficits, since data on these characteristics were not systemically evaluated [15, 21, 22]. Central application of IGF-1 in adult rodents resulted in decreased depression-like behavior, however, no volumetric information was provided in this specific study [23].

With regard to WM, both increases of the axonal volume (physiologically about $46 \%$ of WM) and of myelin sheaths (i.e., oligodendrocytes, physiologically about $24 \%$ of WM) are conceivable [24]. In view of reports on swelling of myelin sheaths in consequence to increased 
IGF-1 levels, and an increase of the myelin content in IGF1-overexpressing mice, expansion of the myelin compartment may explain our observation [22]. Advanced MRI including diffusion tensor imaging and ${ }^{1} \mathrm{H}-\mathrm{MR}$-spectroscopy may be used to assign the volume changes more specifically to a histopathological substrate. With regard to GM, the observed changes may reflect expansion of axons and dendrites of fully differentiated neurons. Experimental histopathological work and theoretical considerations, however, suggest that the glia cell compartment may be an equally important source of macroscopical volume changes [25]. Neurogenesis triggered by IGF-1 and GH, i.e., an increased number of neurons, seems more unlikely as neurogenesis is restricted to few brain regions such as the hippocampus.

In general, decreases of local or global GM or WM volume due to primary neurodegeneration or secondary to inflammatory conditions, neurovascular pathology or toxic agents are found more often than volume increases [26]. We investigated the influence of arterial hypertension and diabetes, the two cardiovascular factors significantly more frequent in the acromegaly group in this sample, finding trend decreases of $\mathrm{GM}_{\text {total }}$ volumes across in association with the presence of these comorbidities across both groups, and no effects $(P>0.10)$ for $\mathrm{WM}_{\text {total }}$ and $\mathrm{BV}_{\text {total }}$. This strengthens that volume effects are unlikely to be driven by cardiovascular comorbidity but are rather specific to acromegaly. Disease specific increases of GM volume are rather exceptional and mostly represented in the spectrum of neurodevelopmental disorders such as hemimegalencephaly or cerebral gigantism. Patients with these disorders often present with general developmental delay or seizures. Also cortical dysplasia may be volumetrically expansive, however is highly focal in most cases [27]. Increases of basal ganglia volumes have been observed as consequence of long-standing neuroleptic drug treatment [28].

The split into patient subgroups with shorter and longer hormone excess allowed to generate a number of new hypotheses: first, no linear relationship between the estimated time of hormone excess and brain size could be observed. Rather, for both GM and WM, the volume increases seem to saturate after about 10 years. Even a slight decrease was seen after 10 years that, however, failed to reach significance. Second, as far as extractable from cross-sectional data, CSF spaces were decreased first, while TIV continued to increase, giving space to CSF that returned to normal absolute values. This pattern may indicate that the brain volume increase preceeds (or overrides) changes of the intracranial vault in the early phase. As some IGF-1/GH effects in acromegaly, for instance on bones, skull and joints, are reversible only at initial stages, but not if the disease is left untreated for several years [1], it remains pivotal to determine if there is also a critical limit up to which cerebral changes are reversible. We also revealed no correlation between the current hormone status and brain volumes, confirming that brain volume changes are long-term effects and unlikely to follow short term fluctuations of IGF-1 (or GH).

Although widespread anatomical systems may be affected by the ubiquitary presence of IGF-1 and GH in the brain, some regions may be more susceptible than others [29]. Clinically, however, a large variety of neuropsychiatric symptoms rather argues for disturbances of multiple circuits, including the regulation of affect and anxiety related behavior, pain perception and higher cognitive functions. It should be mentioned that we do not know yet to which degree the observed structural changes actually affect brain function. Future studies in acromegalic patients are underway to determine if psychopathological or cognitive abnormalities are indeed associated with these morphological changes.

Differences of WML counts were subtle with patients being overrepresented in only one of the higher WML lesion categories. The effect of acromegaly on this simple neurovascular parenchymal marker was into the expected direction, as acromegaly provokes cardiovascular and metabolic complications including arterial hypertension and diabetes. Both diagnoses were indeed more frequent in patients than controls and are known to be associated with increased number of WML [30, 31]. Therefore, the WML finding of this study probably reflects the increased cardiovascular and metabolic comorbidity. In addition, the WML effect was stronger in the subgroup with longer hormone excess, which further concurs with the hypothesis that hypertension and cardiovascular diseases develop as a consequence of chronic alterations of the endothelial and cardiac function in acromegaly [1]. We cannot exclude, however, that IGF-1/GH increase the susceptibility to ischemia by different mechanismus, e.g., by reducing the remyelination potential. In any case, structural damage to the fiber tracts by microangiopathy can add to cognitive decline [32] and makes careful monitoring and treatment of cardiovascular and metabolic comorbidities mandatory.

The study is mainly limited by its cross-sectional design and heterogeneous patient sample with different disease duration. In addition, the method of stratification into shorter and longer duration of hormone excess is debatable. We performed a supportive analysis using linear regression to explore if the estimated time of hormone excess allows correlates with total brain size, yet found no improvement in the model fit compared with the group stratification (data not shown). Further, as the variable is a compound of three other variables (time of diagnosis, estimated 8 years of previous hormone excess and time since biochemical control), its reliability is generally limited. Methodologically, TIV is a 
standard intraindividual reference for brain volume, however, in the case of acromegaly it may not be stable. Despite this, we also analyzed volumetric measures normalized to TIV, as TIV explains a large proportion of the variance of the brain volume. Subtle changes of the group statistics confirmed that TIV covaried with the disease process which underlines the importance to match for other factors cocorrelating with TIV, particularly gender and body height. Furthermore, the calculation of total CSF volume including the external subarachnoid spaces is challenging for automated methods that do not explicitly model extradural tissue. This may in part explain the larger group overlap for analysis of CSF and TIV.

In conclusion, we provide first in vivo evidence of a disturbed macroscopic brain architecture in acromegaly with increases of total GD and WM volumes. These changes seem to occur during the first 10 years of hormone excess at the expense of the CSF compartment, with parallel increases of the total intracranial volume. In addition, a tendency for increased neurovascular pathology is demonstrated, most likely due more frequent metabolic and cardiovascular comorbidities. Future work will need to clarify which brain functions and neuropsychiatric syndromes relate to these changes, and if treatment can reverse them.

Acknowledgments We thank Iris Miedl, Doris Goy-Alsing, Rosa Hemauer, Reinhold Borschke and Elke Schreiter for MRI data acquisition and excellent patient care.

Open Access This article is distributed under the terms of the Creative Commons Attribution Noncommercial License which permits any noncommercial use, distribution, and reproduction in any medium, provided the original author(s) and source are credited.

\section{References}

1. Colao A, Ferone D, Marzullo P, Lombardi G (2004) Systemic complications of acromegaly: epidemiology, pathogenesis, and management. Endocr Rev 25:102-152. doi:10.1210/er.2002-0022

2. Bonapart IE, van Domburg R, ten Have SM, de Herder WW, Erdman RA, Janssen JA et al (2005) The 'bio-assay' quality of life might be a better marker of disease activity in acromegalic patients than serum total IGF-I concentrations. Eur J Endocrinol 152:217-224. doi:10.1530/eje.1.01838

3. Blickenstorfer E (1953) Psychiatric and genealogic study of 51 cases of acromegaly. Schweiz Arch Neurol Psychiatr 71:371-374

4. Blickenstorfer E (1953) Genealogy and psychopathology of 51 acromegaly patients. Acta Endocrinol (Copenh) 13:123-137

5. Blickenstorfer E (1951) Psychiatry and genealogy of acromegaly; studies from the confines of psychopathology of endocrinology. Arch Psychiatr Nervenkr Z Gesamte. Neurol Psychiatr (Bucur) 186:88-122

6. Bleuler M (1951) Personality changes in pituitary disorders. BMJ $1: 580-581$

7. Bleuler M (1951) The psychopathology of acromegaly. J Nerv Ment Dis 113:497-511. doi:10.1097/00005053-195101000-00050
8. Russo VC, Gluckman PD, Feldman EL, Werther GA (2005) The insulin-like growth factor system and its pleiotropic functions in brain. Endocr Rev 26:916-943. doi:10.1210/er.2004-0024

9. Aberg MA, Aberg ND, Palmer TD, Alborn AM, CarlssonSkwirut C, Bang P et al (2003) IGF-I has a direct proliferative effect in adult hippocampal progenitor cells. Mol Cell Neurosci 24:23-40. doi:10.1016/S1044-7431(03)00082-4

10. Schneider HJ, Pagotto U, Stalla GK (2003) Central effects of the somatotropic system. Eur J Endocrinol 149:377-392. doi: 10.1530/eje.0.1490377

11. Ransome MI, Goldshmit Y, Bartlett PF, Waters MJ, Turnley AM (2004) Comparative analysis of CNS populations in knockout mice with altered growth hormone responsiveness. Eur J Neurosci 19:2069-2079. doi:10.1111/j.0953-816X.2004.03308.x

12. Ajo R, Cacicedo L, Navarro C, Sanchez-Franco F (2003) Growth hormone action on proliferation and differentiation of cerebral cortical cells from fetal rat. Endocrinology 144:1086-1097. doi: 10.1210/en.2002-220667

13. O'Kusky JR, Ye P, D'Ercole AJ (2000) Insulin-like growth factor-I promotes neurogenesis and synaptogenesis in the hippocampal dentate gyrus during postnatal development. J Neurosci $20: 8435-8442$

14. Aberg MA, Aberg ND, Hedbacker H, Oscarsson J, Eriksson PS (2000) Peripheral infusion of IGF-I selectively induces neurogenesis in the adult rat hippocampus. J Neurosci 20:2896-2903

15. Beck KD, Powell-Braxton L, Widmer HR, Valverde J, Hefti F (1995) Igf1 gene disruption results in reduced brain size, CNS hypomyelination, and loss of hippocampal granule and striatal parvalbumin-containing neurons. Neuron 14:717-730. doi: 10.1016/0896-6273(95)90216-3

16. Coculescu M (1999) Blood-brain barrier for human growth hormone and insulin-like growth factor-I. J Pediatr Endocrinol Metab 12:113-124

17. van der Lely AJ, Beckers A, Daly AF, Lamberts SWJ, Clemmons DR (2005) Acromegaly: pathology, diagnosis and treatment. Taylor \& Francis, Boca Raton

18. Giustina A, Barkan A, Casanueva FF, Cavagnini F, Frohman L, Ho K et al (2000) Criteria for cure of acromegaly: a consensus statement. J Clin Endocrinol Metab 85:526-529. doi:10.1210/jc. 85.2.526

19. Ashburner J, Friston KJ (2005) Unified segmentation. Neuroimage 26:839-851. doi:10.1016/j.neuroimage.2005.02.018

20. O'Sullivan M, Measurement of brain atrophy in subcortical vasculr disease: A comparison of different approaches and the impact of ischaemic lesions. NeuroImage (in press)

21. D'Ercole AJ, Ye P, O'Kusky JR (2002) Mutant mouse models of insulin-like growth factor actions in the central nervous system. Neuropeptides 36:209-220. doi:10.1054/npep.2002.0893

22. Carson MJ, Behringer RR, Brinster RL, McMorris FA (1993) Insulin-like growth factor I increases brain growth and central nervous system myelination in transgenic mice. Neuron 10:729740. doi:10.1016/0896-6273(93)90173-O

23. Hoshaw BA, Malberg JE, Lucki I (2005) Central administration of IGF-I and BDNF leads to long-lasting antidepressant-like effects. Brain Res 1037:204-208. doi:10.1016/j.brainres.2005.01. 007

24. Miller DH, Barkhof F, Frank JA, Parker GJ, Thompson AJ (2002) Measurement of atrophy in multiple sclerosis: pathological basis, methodological aspects and clinical relevance. Brain 125:16761695. doi:10.1093/brain/awf177

25. Rajkowska G, Miguel-Hidalgo JJ, Wei J, Dilley G, Pittman SD, Meltzer HY et al (1999) Morphometric evidence for neuronal and glial prefrontal cell pathology in major depression. Biol Psychiatry 45:1085-1098. doi:10.1016/S0006-3223(99)00041-4

26. Giannakopoulos P, Hof PR, Michel JP, Guimon J, Bouras C (1997) Cerebral cortex pathology in aging and Alzheimer's 
disease: a quantitative survey of large hospital-based geriatric and psychiatric cohorts. Brain Res Brain Res Rev 25:217-245. doi: 10.1016/S0165-0173(97)00023-4

27. Mathern GW, Andres M, Salamon N, Chandra PS, Andre VM, Cepeda $\mathrm{C}$ et al (2007) A hypothesis regarding the pathogenesis and epileptogenesis of pediatric cortical dysplasia and hemimegalencephaly based on MRI cerebral volumes and NeuN cortical cell densities. Epilepsia 48(Suppl 5):74-78. doi:10.1111/j. 1528-1167.2007.01292.x

28. Corson PW, Nopoulos P, Miller DD, Arndt S, Andreasen NC (1999) Change in basal ganglia volume over 2 years in patients with schizophrenia: typical versus atypical neuroleptics. Am J Psychiatry 156:1200-1204

29. Aberg ND, Brywe KG, Isgaard J (2006) Aspects of growth hormone and insulin-like growth factor-I related to neuroprotection, regeneration, and functional plasticity in the adult brain. ScientificWorldJournal 6:53-80. doi:10.1100/tsw.2006.22

30. Jongen C, van der Grond J, Kappelle LJ, Biessels GJ, Viergever MA, Pluim JP (2007) Automated measurement of brain and white matter lesion volume in type 2 diabetes mellitus. Diabetologia 50:1509-1516. doi:10.1007/s00125-007-0688-y

31. de Leeuw FE, de Groot JC, Oudkerk M, Witteman JC, Hofman A, van Gijn J et al (2002) Hypertension and cerebral white matter lesions in a prospective cohort study. Brain 125:765-772. doi: 10.1093/brain/awf077

32. Smith EE, Gurol ME, Eng JA, Engel CR, Nguyen TN, Rosand J et al (2004) White matter lesions, cognition, and recurrent hemorrhage in lobar intracerebral hemorrhage. Neurology 63:16061612 